\title{
Identificação de plantas com potencial apícola provenientes de sistemas de monitoramento de colmeias no estado de Santa Catarina, Brasil
}

\author{
Identification of plant with beekeeping potential from beehive monitoring systems in the Santa \\ Catarina state, Brazil \\ Identificación de plantas con potencial apícola procedentes de sistemas de monitoreo de colmenas \\ en el estado de Santa Catarina
}

Recebido: 18/05/2021 | Revisado: 23/05/2021 | Aceito: 27/05/2021 | Publicado: 23/06/2021

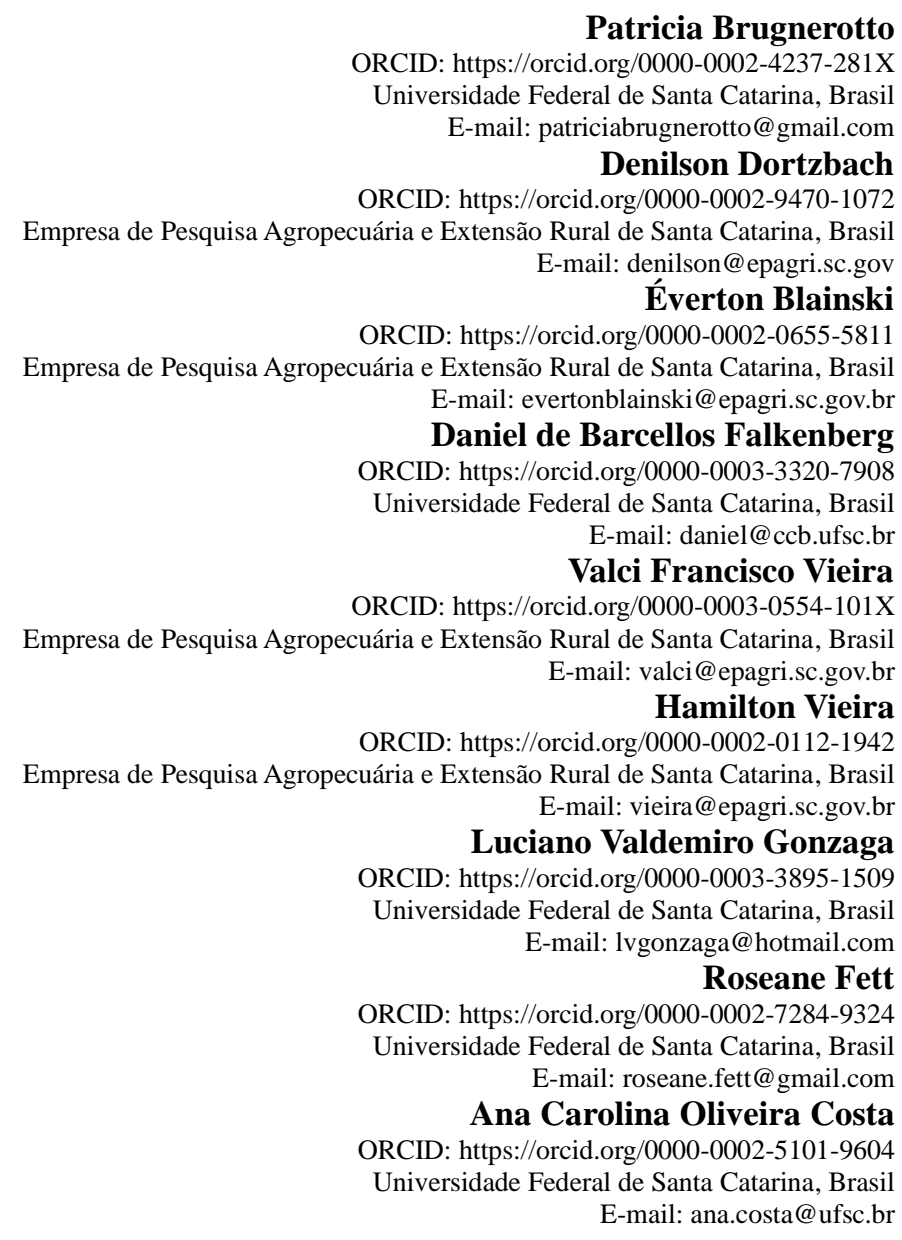

\begin{abstract}
Resumo
A caracterização de espécies vegetais com potencial apícola presentes em sistemas de produção apícola pode trazer muitas vantagens. Entre os benefícios, destaca-se a definição do local de implantação das colmeias nos agroecossistemas de acordo com a disponibilidade de recursos florais, permitindo aumentar a produtividade e qualidade dos méis e demais produtos provenientes das abelhas. Nesse sentido, o objetivo desse trabalho foi coletar espécies de plantas com flores visitadas por abelhas próximas de sistemas de monitoramento de colmeias em seis zonas agroecológicas do estado de Santa Catarina, Brasil, bem como realizar a identificação taxonômica e geográfica destas espécies. Para cada amostra foi registrada sua localização, para estimar a distância do percurso realizado pelas abelhas, uma vez que a localização da colmeia era fixa. Nas 157 amostras identificadas, 81 espécies e 68 gêneros foram encontrados em 33 famílias, sendo Asteraceae e Fabaceae predominantes. Para Asteraceae, os gêneros mais relatados foram Baccharis, Tithonia, Senecio e Sphagneticola, enquanto para Fabaceae, o gênero mais relatado foi Trifolium. Espécies como Sphagneticola trilobata (Asteraceae), Trifolium repens, T. pratense, Cajanus cajan, Vicia sp. (todas Fabaceae), Verbena litoralis, Lantana undulata (ambas Verbenaceae), Raphanus sativus (Brassicaceae) e
\end{abstract}


Clidemia hirta (Melastomataceae) podem ser consideradas interessantes do ponto de vista apícola, pois permaneceram com flores entre o primeiro e último dia de coleta em algumas regiões ( 60 dias). Apesar das distâncias entre as plantas e as colmeias apresentarem elevada variabilidade (8 a $1154 \mathrm{~m})$, geralmente as distâncias não ultrapassavam $750 \mathrm{~m}$.

Palavras-chave: Apicultura; Apis mellifera; Asteraceae; Biodiversidade; Flora apícola; Fabaceae.

\begin{abstract}
The characterization of plant species with beekeeping potential, which are present in beekeeping production systems, can bring many advantages. Among the benefits, the choice of the beehives implantation place according to the availability of floral resources in the agroecosystems, allowing to increase the productivity and the quality of honeys and other products from the bees, are highlighted. In this sense, the aim of this work was to collect flowering plant species, which were visited by bees close to beehive monitoring systems, in six agroecological zones of the Santa Catarina state, Brazil. The taxonomic and geographical identification of these species were also carried out. For each sample, its location was registered in order to estimate the route distance taken by the bees, since the location of the hive was fixed. Of the 157 identified samples, 81 species and 68 genera were found in 33 families, with Asteraceae and Fabaceae being predominant. For Asteraceae, the most reported genera were Baccharis, Tithonia, Senecio, and Sphagneticola, while for Fabaceae, the most reported genus was Trifolium. Species such as Sphagneticola trilobata (Asteraceae), Trifolium repens, T. pratense, Cajanus cajan, Vicia sp. (all Fabaceae), Verbena litoralis, Lantana undulata (both Verbenaceae), Raphanus sativus (Brassicaceae), and Clidemia hirta (Melastomataceae) can be considered interesting from an apicultural point of view, as they remained with flowers between the first and last day of sample collection in some regions ( 60 days). Although the distances between the plants and the beehives have high variability ( 8 to $1154 \mathrm{~m}$ ), the distances generally do not exceed $750 \mathrm{~m}$.
\end{abstract}

Keywords: Beekeeping; Apis mellifera; Asteraceae; Biodiversity; Bee flora; Fabaceae.

\title{
Resumen
}

La caracterización de especies vegetales con potencial apícola presentes en sistemas de producción de mieles puede traer muchas ventajas. Entre los beneficios, se destaca la definición del lugar de implantación de las colmenas en los agroecosistemas de acuerdo con la disponibilidad de recursos florales, permitiendo aumentar la productividad y calidad de las mieles y demás productos procedentes de las abejas. En este sentido, el objetivo del trabajo fue recoger especies de plantas con flores visitadas por abejas cercanas a sistemas de monitoreo de colmenas en seis zonas agroecológicas del estado de Santa Catarina, Brasil, así como realizar la identificación taxonómica y geográfica de estas especies. Para cada muestra se registró su ubicación para estimar la distancia del recorrido por las abejas, ya que la ubicación de la colmena era fija. En las 157 muestras identificadas, 81 especies y 68 géneros fueron encontrados en 33 familias, siendo predominantes Asteraceae y Fabaceae. Para Asteraceae, los géneros más relatados fueron Baccharis, Tithonia, Senecio y Sphagneticola, mientras que para Fabaceae, el género más reportado fue Trifolium. Especies como Sphagneticola trilobata (Asteraceae), Trifolium repens, T. pratense, Cajanus cajan, Vicia sp. (todas Fabaceae), Verbena litoralis, Lantana undulata (ambas Verbenaceae), Raphanus sativus (Brassicaceae) y Clidemia hirta (Melastomataceae) pueden considerarse interesantes desde el punto de vista apícola, ya que permanecieron con flores entre el primer y el último día de recolección en algunas regiones ( 60 días). Aunque las distancias entre las plantas y las colmenas presentan una gran variabilidad (8 a $1154 \mathrm{~m}$ ), generalmente las distancias no pasaban $750 \mathrm{~m}$. Palabras clave: Apicultura; Apis mellifera; Asteraceae; Biodiversidad; Flora apícola; Fabaceae.

\section{Introdução}

Nos últimos anos, a região Sul do Brasil tem se destacado como a principal produtora de mel, responsável por aproximadamente $40 \%$ da produção no país (Abemel, 2020). A apicultura é uma importante atividade para o agronegócio catarinense. O estado de Santa Catarina é líder nacional em produtividade de mel, com aproximadamente $65 \mathrm{~kg} / \mathrm{km}^{2}, 13 \mathrm{vezes}$ superior à média do Brasil, sendo que a safra 2019/2020 de mel atingiu 7,5 mil toneladas. De acordo com Instituto Brasileiro de Geografia e Estatística (Ibge, 2017), em 2017 Santa Catarina também se destacou em $2^{0}$ lugar entre os 27 estados brasileiros por apresentar cerca de 17 mil unidades de estabelecimentos agropecuários com apicultura, os quais continham aproximadamente 300 mil colmeias. Isso demonstra a importância socioeconômica do estado catarinense no contexto apícola nacional. Portanto, além da apicultura ampliar a geração de renda, movimentar a economia local, promover fortalecimento da cadeia produtiva e fornecer inúmeros produtos apícolas, também é uma atividade que tem estreita relação com o desenvolvimento e a conservação da vegetação de uma região. Nesse contexto, a identificação das espécies vegetais visitadas por abelhas assume grande importância. 
A flora apícola, que compõe o pasto ou pastagem apícola, é caracterizada por espécies vegetais que oferecem néctar, pólen ou secreções às abelhas (Almeida, et al., 2003; Camargo, 2002; Coradin, et al., 2011), e esta pode variar em função do tipo de vegetação, das condições edafoclimáticas, das estações do ano e das próprias regiões geográficas (Salomé \& Orth, 2004; Santos, et al., 2006). Portanto, diversas espécies podem ser consideradas plantas apícolas quando estas são visitadas pelas abelhas na procura de recursos nectaríferos e/ou polínicos (Carvalho \& Marchini, 1999).

O estado de Santa Catarina possui rica biodiversidade, com vegetações heterogêneas e diversificadas devido, principalmente, às variações climáticas e geomorfológicas existentes (Santos, et al., 2016). Embora ocorrendo no mesmo estado, algumas espécies podem ser comuns e características de determinada região, enquanto em outra região a mesma espécie pode ser considerada rara. Dessa maneira, o conhecimento da presença de espécies de plantas em determinada região, bem como seus ciclos de florescimento (Salomé, 2002), são de fundamental importância, não somente para conservar e explorar racionalmente as abelhas, mas para identificar, preservar e multiplicar as espécies vegetais mais importantes da região (Carvalho \& Marchini, 1999; Pirani \& Cortopassi-Laurino, 1993).

É importante que apicultores e/ou agentes de assistência técnica conheçam as áreas prioritárias, ou seja, regiões de produção de espécies com potencial apícola e seus períodos de floração para um planejamento adequado de manejo e maximização do aproveitamento destes recursos para a produção de méis diversificados e demais produtos apícolas. Essas ações podem deter a perda de biodiversidade e usar sustentavelmente os recursos naturais em cadeias produtivas e em atividades de subsistência de comunidades humanas, metas que fazem parte do Objetivo 15 das políticas de desenvolvimento sustentável da Organização das Nações Unidas (ONU, 2021). Inúmeras espécies da flora catarinense são conhecidas, principalmente por mérito dos botânicos Raulino Reitz e Roberto Miguel Klein, integrantes do Herbário Barbosa Rodrigues e criadores da Flora Ilustrada Catarinense (Reitz, 1965-1989; Reis, 1996-2013). No entanto, até o presente momento, nenhum estudo investigou a caracterização de floradas com potencial apícola próximas de colmeias monitoradas no Brasil.

No Brasil, a Empresa de Pesquisa Agropecuária e Extensão Rural de Santa Catarina/ Centro de Informações de Recursos Ambientais e de Hidrometeorologia de Santa Catarina (EPAGRI/CIRAM) foi pioneira no desenvolvimento e implementação de um projeto denominado Apis On-line (Epagri, 2021). Esse projeto é composto por estações automatizadas com telemetria que monitoram colmeias e enviam as informações de hora em hora da temperatura e da umidade relativa do ar internas e externas, do peso de produção de mel da colmeia e do volume de precipitação pluviométrica para o banco de dados da EPAGRI. O monitoramento automatizado foi implementado em 2018 em seis zonas agroecológicas do estado de Santa Catarina, as quais compreendiam as cidades de Araranguá, Caçador, Joinville, Mafra, São Joaquim e São Miguel do Oeste. Os locais selecionados representam regiões importantes na criação e preservação das abelhas e com grande capilaridade para atuação dos agentes de assistência técnica. Além disso, o monitoramento automatizado de colmeias promove um grande avanço tecnológico para a apicultura brasileira, devido à possibilidade de monitorar pragas e doenças na colmeia, atividades de difusão e ações de caracterização e monitoramento da oferta de alimentos disponíveis às abelhas e da qualidade do mel e pólen armazenados pelas abelhas.

Nesse sentido, a partir da observação direta de plantas visitadas por abelhas Apis mellifera em seis zonas agroecológicas catarinenses, nas quais estavam alocadas colmeias monitoradas pelo sistema Apis On-line, o objetivo desse estudo foi coletar essas espécies e realizar a identificação taxonômica.

\section{Metodologia}

Foram coletados ramos com folhas e flores de plantas próximas às colmeias monitoradas nas cidades de Araranguá, Caçador, Joinville, Mafra, São Miguel do Oeste e São Joaquim. Nestes locais foram realizadas três coletas no ano de 2018 (outubro a dezembro), com intervalos entre 20 e 30 dias, aproximadamente, e em um raio aproximado de até $1 \mathrm{~km}$ de cada 
colmeia monitorada. A distribuição geográfica das estações de monitoramento e consequentemente as áreas de coleta foram determinadas pelas diferenças climáticas existentes - classificação climática de Köppen (Köppen, 1948) e classificação de zonas agroecológicas do zoneamento agroecológico e socioeconômico do estado de Santa Catarina (Thomé, et al., 1999) considerando-se altitudes de próxima de zero ao nível do mar no litoral de Santa Catarina (Subtropical úmido, Cfa) até próximas de 1400 metros de altitude (Subtropical úmido, Cfb). Na Tabela 1 são apresentadas as principais informações das localizações das colmeias, bem como as datas de coletas das plantas.

Tabela 1. Datas de coleta das plantas e localizações das colmeias monitoradas pelo Apis On-line em 2018.

\begin{tabular}{|c|c|c|c|}
\hline Municípios & Zona Agroecológica $^{\mathrm{a}}$ & Coordenadas geográficas das colmeias & Datas das coletas \\
\hline \multirow{3}{*}{ Araranguá } & 2B - Carbonífera, & Latitude: $28,9333 \mathrm{~S}$ & Coleta 1: 29/10/2018 \\
\hline & Extremo Sul e Colonial & Longitude: 49,4961 W & Coleta 2: 20/11/2018 \\
\hline & Serrana & Altitude: $3 \mathrm{~m}$ & Coleta 3: 20/12/2018 \\
\hline \multirow{3}{*}{ Caçador } & $3 \mathrm{~A}$ - Vale do Rio do & Latitude: $26,8181 \mathrm{~S}$ & Coleta $1: 29 / 10 / 2018$ \\
\hline & Peixe e Planalto Norte & Longitude: 50,9919 W & Coleta 2: 22/11/2018 \\
\hline & Central & Altitude: $934 \mathrm{~m}$ & Coleta 3: 18/12/2018 \\
\hline \multirow{3}{*}{ Joinville } & 1A - Litoral Norte, & Latitude: $26,2047 \mathrm{~S}$ & Coleta $2: 21 / 11 / 2018$ \\
\hline & Vales dos Rios Itajaí e & Longitude: 48,9203 W & Coleta 3: 19/12/2018 \\
\hline & Tijucas & Altitude: $44 \mathrm{~m}$ & Coleta 3: $18 / 12 / 2018$ \\
\hline \multirow{3}{*}{ Mafra } & \multirow{3}{*}{$\begin{array}{c}\text { 3B - Planalto Norte } \\
\text { Catarinense }\end{array}$} & Latitude: $26,1389 \mathrm{~S}$ & Coleta 1: 01/11/2018 \\
\hline & & Longitude: $49,8114 \mathrm{~W}$ & Coleta $2: 22 / 11 / 2018$ \\
\hline & & Altitude $792 \mathrm{~m}$ & Coleta 3: $18 / 12 / 2018$ \\
\hline \multirow{3}{*}{ São Joaquim } & \multirow{3}{*}{$\begin{array}{c}5 \text { - Planalto Serrano de } \\
\text { São Joaquim }\end{array}$} & Latitude: $28,2739 \mathrm{~S}$ & Coleta 1: 29/10/2018 \\
\hline & & Longitude: 49,9339 W & Coleta $2: 20 / 11 / 2018$ \\
\hline & & Altitude: $1206 \mathrm{~m}$ & Coleta 3: 20/12/2018 \\
\hline \multirow{3}{*}{$\begin{array}{c}\text { São Miguel do } \\
\text { Oeste }\end{array}$} & \multirow{3}{*}{$\begin{array}{c}3 \mathrm{C} \text { - Noroeste } \\
\text { Catarinense }\end{array}$} & Latitude: $26,7833 \mathrm{~S}$ & Coleta 1: 30/10/2018 \\
\hline & & Longitude: $53,5131 \mathrm{~W}$ & Coleta $2: 21 / 11 / 2018$ \\
\hline & & Altitude: $622 \mathrm{~m}$ & Coleta 3: 19/12/2018 \\
\hline
\end{tabular}

Legenda: a Classificação do zoneamento agroecológico e socioeconômico do estado de Santa Catarina (Thomé, et al., 1999); S - Sul; W Oeste; $\mathrm{m}$ - metros.

Fonte: Autores.

Nestas áreas foram instaladas estações meteorológicas compostas de painéis solares, baterias, dataloggers e demais sensores ambientais (Figura 1) com aquisição dos dados em intervalos horários e armazenados no datalogger. Essas informações são transmitidas de hora em hora para o banco de dados da EPAGRI/CIRAM em Florianópolis por tecnologia celular General Packet Radio Services (GPRS). 
Figura 1. Estações automatizadas e com telemetrias para monitoramento de dados da colmeia e do ambiente.
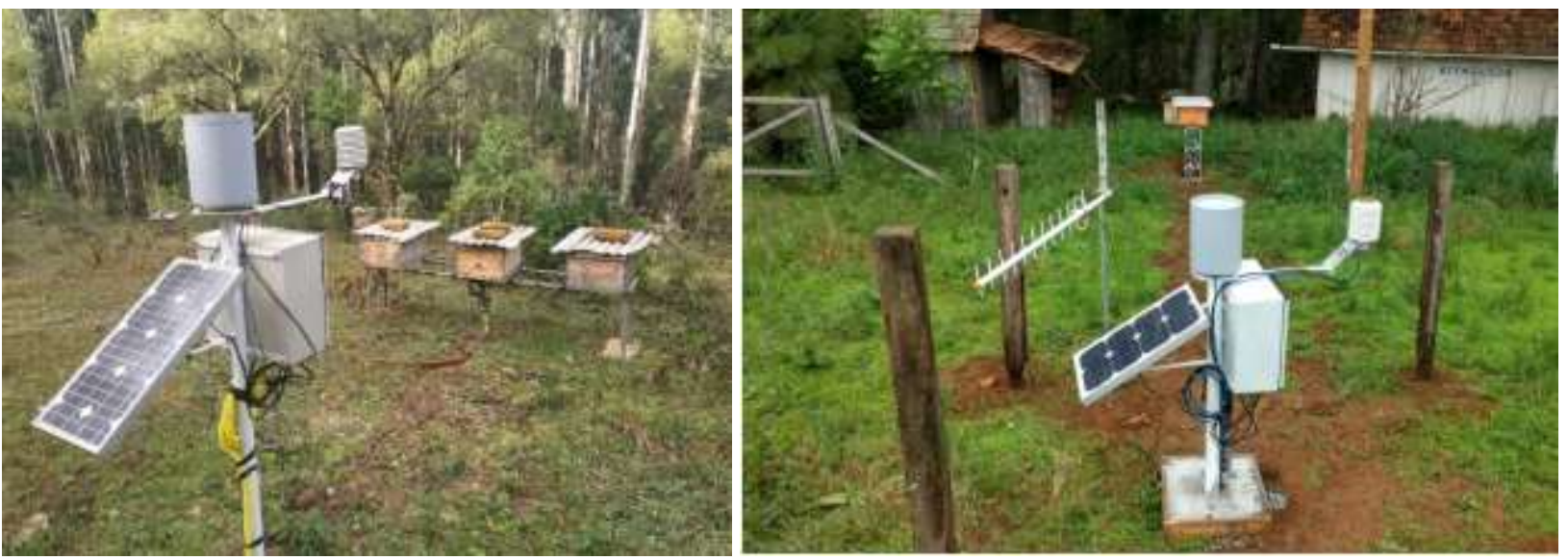

Fonte: Autores e Empresa de Pesquisa Agropecuária e Extensão Rural de Santa Catarina/Centro de Informações de Recursos Ambientais e de Hidrometeorologia de Santa Catarina.

Coordenadas geográficas (latitude e longitude) também foram registradas para cada amostra de planta por meio de um aparelho digital de localização (GPS - Global Positioning System). Para espacialização e cálculos das distâncias em metros dos pontos coletados até cada colmeia, foi utilizado um software $\left(\operatorname{ArcGis}^{\circledR}\right.$ 10.8) de sistema de informações geográficas da Environmental Systems Research Institute - ESRI (Redlands, Califórnia, Estados Unidos da América). Após as coletas, as amostras de plantas foram acondicionadas em embalagens e transportadas ao Centro de Ciências Agrárias (CCA), Departamento de Ciência e Tecnologia de Alimentos (CTA), Laboratório de Química de Alimentos, da Universidade Federal de Santa Catarina (UFSC).

A identificação taxonômica das amostras foi realizada no Departamento de Botânica da UFSC por meio da metodologia tradicional (Stuessy, 2009), que consistiu na comparação das plantas coletadas com exsicatas (Herbário FLOR da UFSC e splink.org.br) e literatura especializada (Reitz, 1965-1989; Souza \& Lorenzi, 2019). Para auxiliar nas identificações, também foram utilizadas imagens registradas durante as coletas.

\section{Resultados e Discussão}

O número de amostras coletadas foi apresentado na Figura 2. Os resultados representam o total de amostras coletadas nas três coletas programadas para cada zona agroecológica. As regiões delimitadas neste estudo que apresentaram maior riqueza de espécies floridas coletadas foram o Extremo Sul (Araranguá) e Planalto Serrano (São Joaquim). 
Figura 2. Número de amostras coletadas próximas de colmeias monitoradas em seis zonas agroecológicas do estado de Santa Catarina.

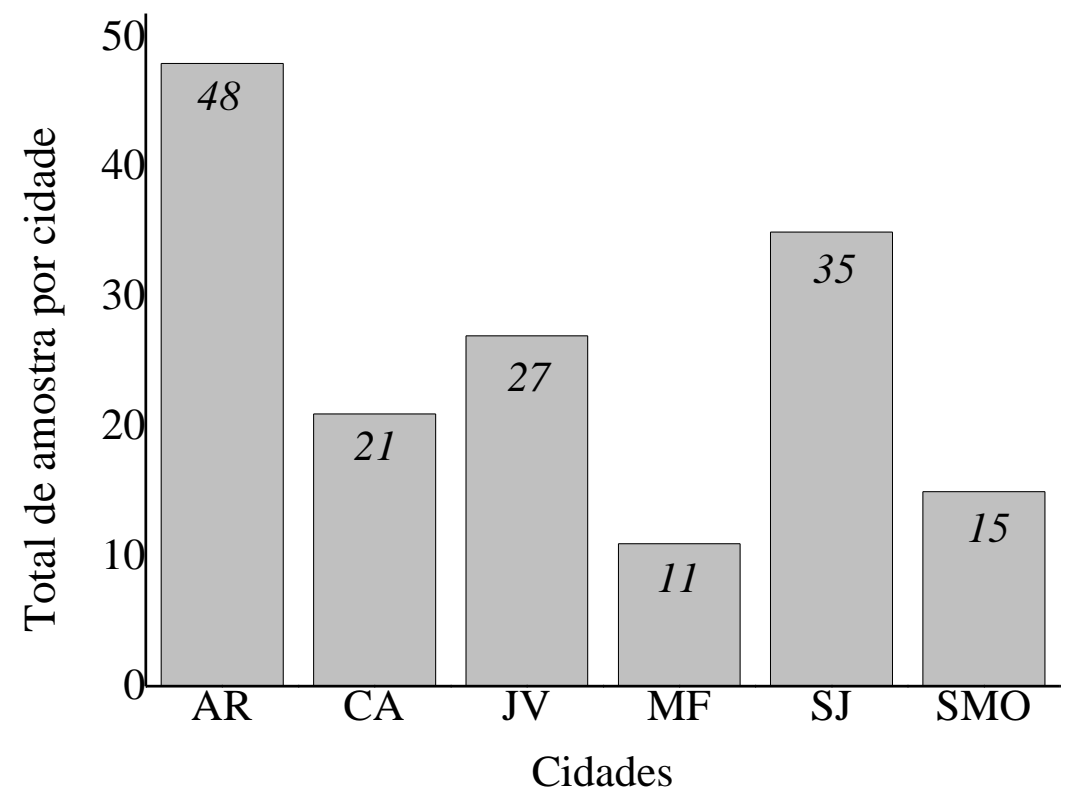

Legenda: Total de amostras coletadas nas proximidades das colmeias monitoradas em Araranguá (AR), Caçador (CA), Joinville (JV), Mafra (MF), São Joaquim (SJ) e São Miguel do Oeste (SMO).

Fonte: Autores.

A identificação das plantas com potencial apícola provenientes dos locais delimitados nesse estudo foi apresentada na Tabela 2. Foram identificadas 33 famílias ( $n=157$ amostras), sendo elas: Amaranthaceae $(n=1)$, Amaryllidaceae $(n=1)$, Anacardiaceae $(n=3)$, Apiaceae $(n=1)$, Apocynaceae $(n=1)$, Arecaceae $(n=2)$, Asteraceae $(n=34)$, Bignoniaceae $(n=2)$, Brassicaceae $(n=14)$, Fabaceae $(n=37)$, Geraniaceae $(n=1)$, Lamiaceae $(n=7)$, Lythraceae $(n=2)$, Marantaceae $(n=1)$, Melastomataceae $(n=6)$, Musaceae $(n=1)$, Myrtaceae $(n=6)$, Oleaceae $(n=3)$, Onagraceae $(n=2)$, Orobanchaceae $(n=1)$, Plantaginaceae ( $n=1)$, Plumbaginaceae $(n=1)$, Rhamnaceae $(n=4)$, Rosaceae $(n=4)$, Rubiaceae $(n=1)$, Rutaceae $(n=1)$, Solanaceae $(n=4)$, Talinaceae $(n=1)$, Tropaeolaceae $(n=2)$,_Urticaceae $(n=1)$, Verbenaceae $(n=9)$, Vitaceae $(n=1)$ e Zingiberaceae $(n=1)$. Destas, Asteraceae e Fabaceae abrangeram juntas $45,5 \%$ do total de amostras encontradas no levantamento. Em relação à riqueza de espécies nas regiões estudadas que totalizou 81 espécies em 68 gêneros, as principais famílias também foram Asteraceae com 15 espécies (13 gêneros) e Fabaceae com 13 espécies (10 gêneros). Com exceção das plantas provenientes de São Miguel do Oeste e Mafra, essas famílias estavam com maior riqueza em todas as demais regiões nesse estudo. Em relação à origem, 40 espécies são exóticas em Santa Catarina, caracterizadas como cultivadas, invasoras, naturalizadas ou ruderais (Souza \& Lorenzi, 2019). Apenas 32 espécies são nativas de Santa Catarina (15 florestais, 10 campestres e 7 indefinidas), e 9 não puderam ter sua origem determinada (Tabela 2). 
Tabela 2. Táxons identificados, origem e ocorrência de plantas apícolas de seis locais do estado de Santa Catarina.

\begin{tabular}{|c|c|c|c|c|c|c|c|c|}
\hline Família & Espécie & $\mathbf{A R}$ & $\mathbf{C A}$ & JV & MF & SJ & SMO & Origem \\
\hline Amaranthaceae & Iresine diffusa & & & & & & $\mathrm{x}$ & ND \\
\hline Amaryllidaceae & Zephyranthes flavissima & & & & & $\mathrm{x}$ & & $\mathrm{N}$ \\
\hline Anacardiaceae & Schinus terebinthifolius & $\mathrm{x}$ & & & $\mathrm{x}$ & & & $\mathrm{N}$ \\
\hline Apiaceae & Foeniculum vulgare & & & & & & $\mathrm{x}$ & $\mathrm{E}$ \\
\hline Apocynaceae & Tabernaemontana catharinensis & $\mathrm{x}$ & & & & & & $\mathrm{N}$ \\
\hline \multirow{2}{*}{ Arecaceae } & Butia eriospatha & & $\mathrm{x}$ & & & & & $\mathrm{N}$ \\
\hline & Roystonea oleracea & $\mathrm{x}$ & & & & & & $\mathrm{E}$ \\
\hline \multirow{15}{*}{ Asteraceae } & Baccharis semiserrata & & $\mathrm{x}$ & & $\mathrm{x}$ & & & $\mathrm{N}$ \\
\hline & Baccharis uncinella & & & & & $\mathrm{x}$ & & $\mathrm{N}$ \\
\hline & Baccharis sp. & & $\mathrm{x}$ & & & $\mathrm{x}$ & & $\mathrm{N}$ \\
\hline & Bidens sp. & & $\mathrm{x}$ & & & & & $\mathrm{E}$ \\
\hline & Cirsium vulgare & & & & $\mathrm{x}$ & & & $\mathrm{E}$ \\
\hline & Conyza sp. & & & $\mathrm{x}$ & & & & ND \\
\hline & Coreopsis tinctoria & & & & $\mathrm{x}$ & & & $\mathrm{E}$ \\
\hline & Helianthus annuus & $\mathrm{x}$ & & & & & & $\mathrm{E}$ \\
\hline & Hypochaeris sp. & & & & & $\mathrm{x}$ & & ND \\
\hline & Leucanthemum vulgare & & & & & $\mathrm{x}$ & & $\mathrm{E}$ \\
\hline & Mikania sp. & $\mathrm{x}$ & & & & & & $\mathrm{N}$ \\
\hline & Senecio brasiliensis & & $\mathrm{x}$ & & $\mathrm{x}$ & $\mathrm{x}$ & & $\mathrm{N}$ \\
\hline & Sphagneticola trilobata & $\mathrm{x}$ & & $\mathrm{x}$ & & & & $\mathrm{E}$ \\
\hline & Tithonia diversifolia & $\mathrm{x}$ & & $\mathrm{x}$ & & & & $\mathrm{E}$ \\
\hline & Vernonia sp. & & & & & $\mathrm{x}$ & & $\mathrm{N}$ \\
\hline Bignoniaceae & Handroanthus chrysotrichus & $\mathrm{x}$ & & & & & & $\mathrm{N}$ \\
\hline \multirow{2}{*}{ Brassicaceae } & Brassica napus & $\mathrm{x}$ & & & & & & $\mathrm{E}$ \\
\hline & Raphanus sativus & $\mathrm{x}$ & $\mathrm{x}$ & & & $\mathrm{x}$ & $\mathrm{x}$ & $\mathrm{E}$ \\
\hline \multirow{13}{*}{ Fabaceae } & Arachis pintoi & $\mathrm{x}$ & & & & & $\mathrm{x}$ & $\mathrm{E}$ \\
\hline & Cajanus cajan & $\mathrm{x}$ & & & & & & $\mathrm{E}$ \\
\hline & Cassia fistula & $\mathrm{x}$ & & & & & & $\mathrm{E}$ \\
\hline & Delonix regia & $\mathrm{x}$ & & $\mathrm{x}$ & & & & $\mathrm{E}$ \\
\hline & Inga luschnathiana & & & $\mathrm{x}$ & & & & $\mathrm{N}$ \\
\hline & Inga sp. & & $\mathrm{x}$ & & & & & $\mathrm{N}$ \\
\hline & Mimosa pudica & & & $\mathrm{x}$ & & & & $\mathrm{N}$ \\
\hline & Schizolobium parahyba & $\mathrm{x}$ & & & & & & $\mathrm{N}$ \\
\hline & Tipuana tipu & $\mathrm{x}$ & & & & & & $\mathrm{E}$ \\
\hline & Trifolium pratense & $\mathrm{x}$ & & & & $\mathrm{x}$ & & $\mathrm{E}$ \\
\hline & Trifolium repens & $\mathrm{x}$ & $\mathrm{x}$ & & & $\mathrm{x}$ & & $\mathrm{E}$ \\
\hline & Trifolium sp. & $\mathrm{x}$ & & & & & & $\mathrm{E}$ \\
\hline & Vicia sp. & $\mathrm{x}$ & $\mathrm{x}$ & & & & & ND \\
\hline Geraniaceae & Pelargonium sp. & & & & & & $\mathrm{x}$ & $\mathrm{E}$ \\
\hline \multirow{4}{*}{ Lamiaceae } & Leonurus sibiricus & $\mathrm{x}$ & & & $\mathrm{x}$ & & & $\mathrm{E}$ \\
\hline & Marsypianthes chamaedrys & $\mathrm{x}$ & & $\mathrm{x}$ & & & & ND \\
\hline & Ocimum basilicum & $\mathrm{x}$ & & & & & & $\mathrm{E}$ \\
\hline & Stachys arvensis & & & & & $\mathrm{x}$ & & $\mathrm{E}$ \\
\hline \multirow{2}{*}{ Lythraceae } & Cuphea hyssopifolia & & & $\mathrm{x}$ & & & & $\mathrm{E}$ \\
\hline & Cuphea sp. & $\mathrm{x}$ & & & & & & $\mathrm{N}$ \\
\hline Marantaceae & Maranta sp. & & & & & & $\mathrm{x}$ & $E$ \\
\hline Melastomataceae & Clidemia hirta & & & $\mathrm{x}$ & & & & $\mathrm{N}$ \\
\hline
\end{tabular}




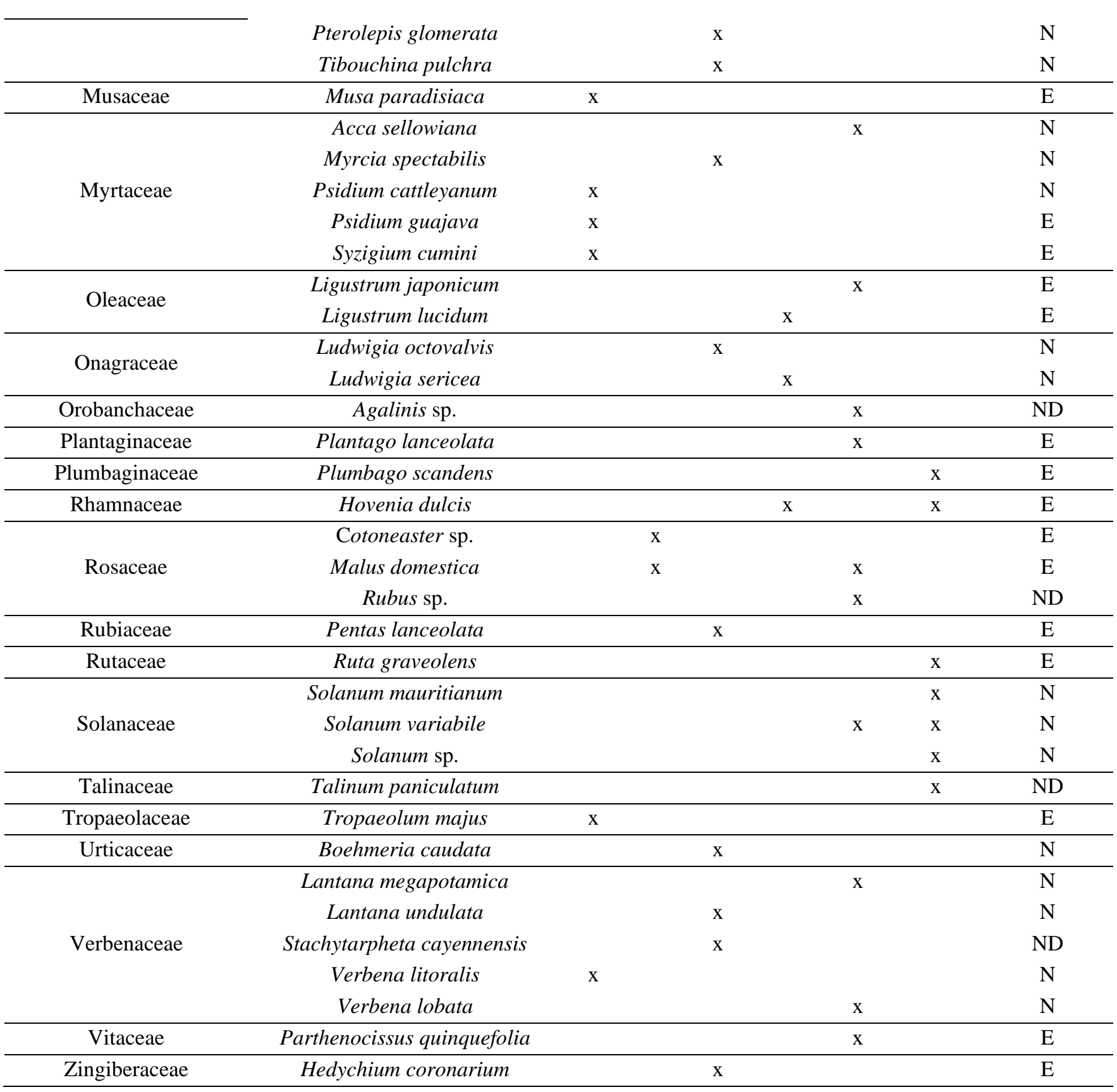

Legenda: AR - Araranguá, CA - Caçador, JV - Joinville, MF - Mafra, SJ - São Joaquim e SMO - São Miguel do Oeste; E - exótica na região; $\mathrm{N}$ - nativa na região; ND - não determinada.

Fonte: Autores.

Nesse estudo, para Asteraceae, os gêneros Baccharis, Senecio, Sphagneticola e Tithonia foram os mais relatados. Enquanto para Fabaceae, os gêneros mais relatados foram Trifolium e Vicia. Ambas famílias possuem morfologias variadas e apresentam ampla distribuição geográfica ocupando quase todos os habitats e formações vegetacionais (Coradin et al., 2011; Souza \& Lorenzi, 2019; Vibrans, et al, 2013). Raphanus sativus (Brassicaceae), conhecido popularmente como nabo forrageiro, representou 8,3\% do total de amostras identificadas (Tabela 3), estando presente nas áreas de estudo de Araranguá, Caçador, São Miguel do Oeste e São Joaquim. Sua presença em diferentes regiões geográficas de Santa Catarina pode estar associada à capacidade adaptativa de brassicáceas às variações agroclimáticas (Sá, 2005). 
Research, Society and Development, v. 10, n. 7, e2710716254, 2021

(CC BY 4.0) | ISSN 2525-3409 | DOI: http://dx.doi.org/10.33448/rsd-v10i7.16254

Tabela 3. Identificação taxonômica e localização geográfica de cada amostra de planta proveniente de seis locais do estado de Santa Catarina

\begin{tabular}{|c|c|c|c|c|c|c|}
\hline \multicolumn{7}{|c|}{ ARARANGUÁ } \\
\hline \multirow[b]{2}{*}{ Famílias } & \multicolumn{2}{|c|}{ Coleta 1} & \multicolumn{2}{|c|}{ Coleta 2} & \multicolumn{2}{|c|}{ Coleta 3} \\
\hline & Espécies & $\begin{array}{c}\text { Distância entre a } \\
\text { espécie e a colmeia (m) }\end{array}$ & Espécies & $\begin{array}{c}\text { Distância entre a espécie } \\
\text { e a colmeia (m) }\end{array}$ & Espécies & $\begin{array}{c}\text { Distância entre a espécie e } \\
\text { a colmeia (m) }\end{array}$ \\
\hline Anacardiaceae & $\begin{array}{c}\text { Schinus } \\
\text { terebinthifolius } \\
\end{array}$ & 374 & Schinus terebinthifolius & 368 & - & - \\
\hline Apocynaceae & $\begin{array}{l}\text { Tabernaemontana } \\
\text { catharinensis }\end{array}$ & 647 & - & - & - & - \\
\hline Arecaceae & Roystonea oleracea & 1108 & - & - & - & - \\
\hline \multirow{3}{*}{ Asteraceae } & $\begin{array}{c}\text { Sphagneticola } \\
\text { trilobata }\end{array}$ & 368 & Sphagneticola trilobata & 327 & $\begin{array}{c}\text { Sphagneticola } \\
\text { trilobata }\end{array}$ & 216 \\
\hline & Mikania sp. & 461 & Tithonia diversifolia & 454 & Tithonia diversifolia & 483 \\
\hline & - & - & Helianthus annuus & 86 & - & - \\
\hline Bignoniaceae & $\begin{array}{l}\text { Handroanthus } \\
\text { chrysotrichus }\end{array}$ & 216 & $\begin{array}{l}\text { Handroanthus } \\
\text { chrysotrichus }\end{array}$ & 220 & - & - \\
\hline \multirow{2}{*}{ Brassicaceae } & Raphanus sativus & 204 & Raphanus sativus & 189 & - & - \\
\hline & - & - & Brassica napus & 516 & - & - \\
\hline \multirow{7}{*}{ Fabaceae } & Tipuana tipu & 189 & Trifolium pratense & 218 & Cassia fistula & 226 \\
\hline & Vicia sp. & 482 & Vicia sp. & 188 & - & - \\
\hline & Trifolium sp. & 204 & Delonix regia & 223 & Delonix regia & 234 \\
\hline & Cajanus cajan & 277 & Cajanus cajan & 460 & Cajanus cajan & 279 \\
\hline & Arachis pintoi & 454 & Arachis pintoi & 460 & Arachis pintoi & 457 \\
\hline & Trifolium repens & 378 & Trifolium repens & 197 & Trifolium repens & 341 \\
\hline & $\begin{array}{l}\text { Schizolobium } \\
\text { parahyba }\end{array}$ & 466 & - & - & - & - \\
\hline \multirow[b]{2}{*}{ Lamiaceae } & - & - & Ocimum basilicum & 134 & Leonurus sibiricus & 290 \\
\hline & - & - & $\begin{array}{l}\text { Marsypianthes } \\
\text { chamaedrys }\end{array}$ & 169 & - & - \\
\hline Lythraceae & Cuphea sp. & 211 & - & - & - & - \\
\hline Musaceae & - & - & Musa paradisiaca & 290 & - & - \\
\hline
\end{tabular}


Research, Society and Development, v. 10, n. 7, e2710716254, 2021

(CC BY 4.0) | ISSN 2525-3409 | DOI: http://dx.doi.org/10.33448/rsd-v10i7.16254

\begin{tabular}{|c|c|c|c|c|c|c|}
\hline \multirow[t]{2}{*}{ Myrtaceae } & $\begin{array}{c}\text { Psidium } \\
\text { cattleyanum }\end{array}$ & 444 & Syzigium cumini & 183 & - & - \\
\hline & Psidium guajava & 462 & Psidium guajava & 182 & - & - \\
\hline Tropaeolaceae & Tropaeolum majus & 185 & Tropaeolum majus & 270 & - & - \\
\hline Verbenaceae & Verbena litoralis & 358 & Verbena litoralis & 189 & Verbena litoralis & 451 \\
\hline \multicolumn{7}{|c|}{ CAÇADOR } \\
\hline & \multicolumn{2}{|c|}{ Coleta 1} & \multicolumn{2}{|c|}{ Coleta 2} & \multicolumn{2}{|c|}{ Coleta 3} \\
\hline Famílias & Espécies & $\begin{array}{c}\text { Distância entre a } \\
\text { espécie e a colmeia (m) }\end{array}$ & Espécies & $\begin{array}{c}\text { Distância entre a espécie } \\
\text { e a colmeia (m) }\end{array}$ & Espécies & $\begin{array}{c}\text { Distância entre a espécie e } \\
\text { a colmeia }(\mathbf{m})\end{array}$ \\
\hline Arecaceae & - & $-\quad-$ & - & $-\quad-$ & Butia eriospatha & 41 \\
\hline \multirow{4}{*}{ Asteraceae } & Senecio brasiliensis & 127 & Senecio brasiliensis & 35 & - & - \\
\hline & Baccharis sp. & 118 & Baccharis sp. & 32 & - & - \\
\hline & $\begin{array}{l}\text { Baccharis } \\
\text { semiserrata }\end{array}$ & 119 & Baccharis semiserrata & 82 & - & - \\
\hline & Bidens sp. & 853 & - & - & - & - \\
\hline Brassicaceae & Raphanus sativus & 722 & Raphanus sativus & 48 & Raphanus sativus & 23 \\
\hline \multirow[b]{2}{*}{ Fabaceae } & Trifolium repens & 46 & Trifolium repens & 27 & Trifolium repens & 11 \\
\hline & $\begin{array}{l}\text { Vicia } \mathrm{sp} . \\
\text { Inga } \mathrm{sp} .\end{array}$ & $\begin{array}{c}55 \\
151\end{array}$ & $\begin{array}{c}\text { Vicia } \mathrm{sp} . \\
- \\
-\end{array}$ & $\begin{array}{c}25 \\
- \\
-\end{array}$ & $\begin{array}{l}\text { Vicia sp. } \\
\text { Inga } \mathrm{sp} .\end{array}$ & $\begin{array}{c}1154 \\
25\end{array}$ \\
\hline Rosaceae & Malus domestica & 17 & Cotoneaster sp. & 69 & - & - \\
\hline \multicolumn{7}{|c|}{ JOINVILLE } \\
\hline \multirow[b]{2}{*}{ Famílias } & \multicolumn{2}{|c|}{ Coleta 1} & \multicolumn{2}{|c|}{ Coleta 2} & \multicolumn{2}{|r|}{ Coleta 3} \\
\hline & Espécies & $\begin{array}{c}\text { Distância entre a } \\
\text { espécie e a colmeia (m) }\end{array}$ & Espécies & $\begin{array}{c}\text { Distância entre a espécie } \\
\text { e a colmeia (m) }\end{array}$ & Espécies & $\begin{array}{c}\text { Distância entre a espécie e } \\
\text { a colmeia (m) }\end{array}$ \\
\hline \multirow[b]{2}{*}{ Asteraceae } & Conyza sp. & 94 & Tithonia diversifolia & 132 & Tithonia diversifolia & 129 \\
\hline & $\begin{array}{c}\text { Sphagneticola } \\
\text { trilobata }\end{array}$ & 77 & Sphagneticola trilobata & 36 & $\begin{array}{c}\text { Sphagneticola } \\
\text { trilobata }\end{array}$ & 8 \\
\hline \multirow{3}{*}{ Fabaceae } & - & - & Inga luschnathiana & 159 & Inga luschnathiana & 160 \\
\hline & - & - & - & - & Delonix regia & 179 \\
\hline & - & - & - & - & Mimosa pudica & 65 \\
\hline Lamiaceae & $\begin{array}{l}\text { Marsypianthes } \\
\text { chamaedrys }\end{array}$ & 96 & - & - & 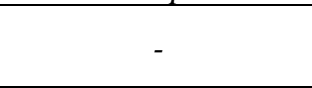 & - \\
\hline
\end{tabular}




\begin{tabular}{|c|c|c|c|c|c|c|}
\hline Lythraceae & - & - & - & - & Cuphea hyssopifolia & 242 \\
\hline \multirow{3}{*}{ Melastomataceae } & Clidemia hirta & 85 & Clidemia hirta & 22 & Clidemia hirta & 11 \\
\hline & - & - & Tibouchina pulchra & 75 & Tibouchina pulchra & 103 \\
\hline & - & - & - & - & Pterolepis glomerata & 56 \\
\hline Myrtaceae & & & & & Myrcia spectabilis & 60 \\
\hline Onagraceae & - & - & - & - & Ludwigia octovalvis & 55 \\
\hline Rubiaceae & - & - & - & - & Pentas lanceolata & 242 \\
\hline Urticaceae & - & - & Boehmeria caudata & 523 & - & - \\
\hline \multirow[b]{2}{*}{ Verbenaceae } & Lantana undulata & 76 & Lantana undulata & 39 & Lantana undulata & 23 \\
\hline & 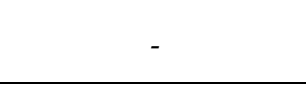 & - & 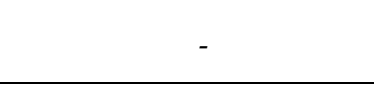 & - & $\begin{array}{c}\text { Stachytarpheta } \\
\text { cayennensis }\end{array}$ & 19 \\
\hline Zingiberaceae & - & - & - & - & $\begin{array}{l}\text { Hedychium } \\
\text { coronarium }\end{array}$ & 21 \\
\hline \multicolumn{7}{|c|}{ MAFRA } \\
\hline \multirow[b]{2}{*}{ Famílias } & \multicolumn{2}{|c|}{ Coleta 1} & \multicolumn{2}{|c|}{ Coleta 2} & \multicolumn{2}{|c|}{ Coleta 3} \\
\hline & Espécies & $\begin{array}{c}\text { Distância entre a } \\
\text { espécie e a colmeia (m) }\end{array}$ & Espécies & $\begin{array}{c}\text { Distância entre a } \\
\text { espécie e a colmeia } \\
(\mathbf{m})\end{array}$ & Espécies & $\begin{array}{c}\text { Distância entre a espécie e } \\
\text { a colmeia (m) }\end{array}$ \\
\hline Anacardiaceae & - & - & Schinus terebinthifolius & 67 & - & - \\
\hline \multirow[t]{2}{*}{ Asteraceae } & $\begin{array}{c}\text { Baccharis } \\
\text { semiserrata }\end{array}$ & 430 & Cirsium vulgare & 69 & Coreopsis tinctoria & 57 \\
\hline & Senecio brasiliensis & 436 & Senecio brasiliensis & 427 & - & - \\
\hline Lamiaceae & - & - & Leonurus sibiricus & 69 & Leonurus sibiricus & 34 \\
\hline Oleaceae & - & - & - & - & Ligustrum lucidum & 110 \\
\hline Onagraceae & - & - & - & - & Ludwigia sericea & 196 \\
\hline Rhamnaceae & - & - & - & - & Hovenia dulcis & 64 \\
\hline \multicolumn{7}{|c|}{ SÃO JOAQUIM } \\
\hline \multirow[b]{2}{*}{ Famílias } & \multicolumn{2}{|c|}{ Coleta 1} & \multicolumn{2}{|c|}{ Coleta 2} & \multicolumn{2}{|c|}{ Coleta 3} \\
\hline & Espécies & $\begin{array}{c}\text { Distância entre a } \\
\text { espécie e a colmeia (m) }\end{array}$ & Espécies & $\begin{array}{l}\text { istância entre a espécie } \\
\text { e a colmeia (m) }\end{array}$ & Espécies & $\begin{array}{c}\text { Distância entre a espécie e } \\
\text { a colmeia (m) } \\
\end{array}$ \\
\hline Amaryllidaceae & - & - & - & - & Zephyranthes flavissima & 253 \\
\hline \multirow{4}{*}{ Asteraceae } & $\begin{array}{l}\text { Leucanthemum } \\
\text { vulgare }\end{array}$ & 510 & $\begin{array}{c}\text { Leucanthemum } \\
\text { vulgare }\end{array}$ & 506 & Hypochaeris sp. & 29 \\
\hline & Senecio brasiliensis & 45 & Senecio brasiliensis & 506 & Vernonia sp. & 207 \\
\hline & Baccharis uncinella & 27 & Baccharis uncinella & 30 & - & - \\
\hline & - & - & Baccharis sp. & 117 & - & - \\
\hline
\end{tabular}




\begin{tabular}{|c|c|c|c|c|c|c|}
\hline \multirow{5}{*}{ Brassicaceae } & Raphanus sativus & 65 & Raphanus sativus & 123 & Raphanus sativus & 39 \\
\hline & Raphanus sativus & 110 & - & - & - & - \\
\hline & Raphanus sativus & 107 & - & - & - & - \\
\hline & Raphanus sativus & 477 & - & - & - & - \\
\hline & Raphanus sativus & 107 & - & - & - & - \\
\hline \multirow{2}{*}{ Fabaceae } & Trifolium pratense & 510 & Trifolium pratense & 520 & Trifolium pratense & 198 \\
\hline & Trifolium repens & 45 & Trifolium repens & 86 & Trifolium repens & 33 \\
\hline Lamiaceae & Stachys arvensis & 64 & - & - & - & - \\
\hline Myrtaceae & - & - & - & - & Acca sellowiana & 247 \\
\hline Oleaceae & $\begin{array}{l}\text { Ligustrum } \\
\text { japonicum }\end{array}$ & 33 & - & - & Ligustrum japonicum & 34 \\
\hline Orobanchaceae & Agalinis sp. & 107 & - & - & - & - \\
\hline Plantaginaceae & - & - & - & - & Plantago lanceolata & 43 \\
\hline \multirow{2}{*}{ Rosaceae } & Rubus sp. & 516 & - & - & - & - \\
\hline & Malus domestica & 66 & - & - & - & - \\
\hline Solanaceae & - & - & - & - & Solanum variabile & 137 \\
\hline Verbenaceae & $\begin{array}{c}\text { Lantana } \\
\text { megapotamica }\end{array}$ & 22 & Verbena lobata & 132 & - & - \\
\hline Vitaceae & - & - & - & - & Parthenocissus quinquefolia & 34 \\
\hline \multicolumn{7}{|c|}{ SÃO MIGUEL DO OESTE } \\
\hline \multirow[b]{2}{*}{ Famílias } & \multicolumn{2}{|c|}{ Coleta 1} & \multicolumn{2}{|c|}{$\begin{array}{c}\text { Coleta } 2 \\
\end{array}$} & \multicolumn{2}{|c|}{ Coleta 3} \\
\hline & Espécies & $\begin{array}{c}\text { Distância entre a } \\
\text { espécie e a colmeia (m) }\end{array}$ & Espécies & $\begin{array}{c}\text { Distância entre a espécie } \\
\text { e a colmeia (m) }\end{array}$ & Espécies & $\begin{array}{c}\text { Distância entre a espécie e } \\
\text { a colmeia (m) }\end{array}$ \\
\hline Apiaceae & - & - & Foeniculum vulgare & 708 & - & - \\
\hline Amaranthaceae & - & - & - & - & Iresine diffusa & 626 \\
\hline Brassicaceae & - & - & Raphanus sativus & 721 & - & - \\
\hline Fabaceae & - & - & - & - & Arachis pintoi & 650 \\
\hline Geraniaceae & - & - & - & - & Pelargonium sp. & 668 \\
\hline
\end{tabular}


Research, Society and Development, v. 10, n. 7, e2710716254, 2021

(CC BY 4.0) | ISSN 2525-3409 | DOI: http://dx.doi.org/10.33448/rsd-v10i7.16254

\begin{tabular}{|c|c|c|c|c|c|c|}
\hline Marantaceae & - & - & - & - & Maranta sp. & 639 \\
\hline Plumbaginaceae & - & - & Plumbago scandens & 830 & - & - \\
\hline Rhamnaceae & Hovenia dulcis & 337 & Hovenia dulcis & 226 & Hovenia dulcis & 222 \\
\hline Rutaceae & - & - & - & - & Ruta graveolens & 688 \\
\hline \multirow{3}{*}{ Solanaceae } & - & - & - & - & Solanum mauritianum & 727 \\
\hline & - & - & - & - & Solanum sp. & 622 \\
\hline & - & - & - & - & Solanum variabile & 626 \\
\hline Talinaceae & - & - & - & - & Talinum paniculatum & 651 \\
\hline
\end{tabular}

Fonte: Autores.

Em relação à contribuição das espécies para o setor apícola catarinense, as origens botânicas de muitos méis catarinenses avaliados por Bergamo (2019) e Silva (2016) -

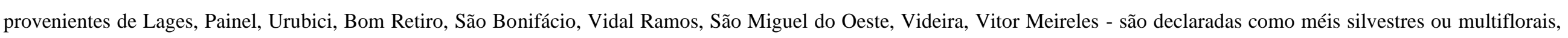

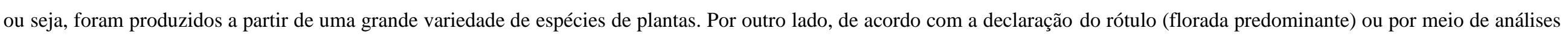

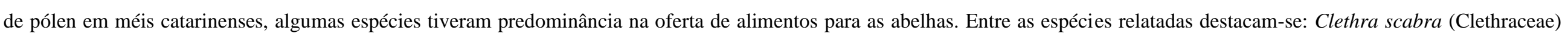

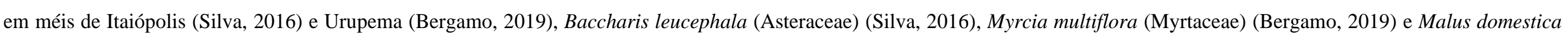

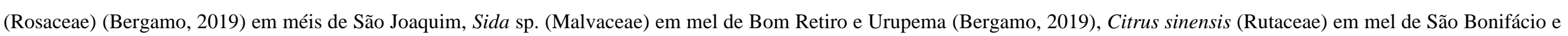

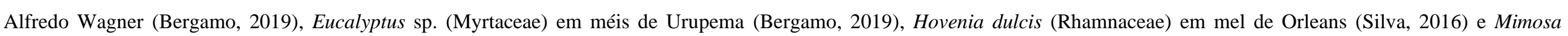

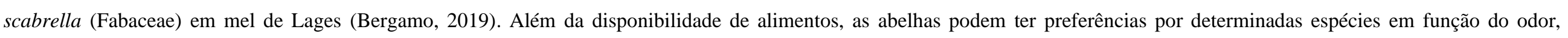

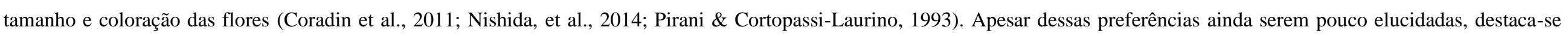

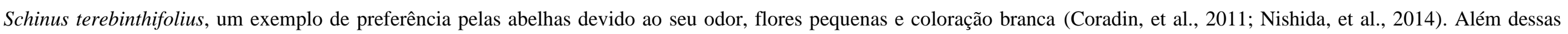

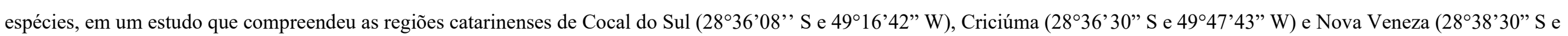
49²1’54” W), 22 famílias (64 espécies) foram visitadas por abelhas, sendo Asteraceae predominante por também apresentar maior número de espécies (Silva, 2005) 
A flora apícola tem influência direta nas características sensoriais e físico-químicas dos méis, sendo estas sujeitas à preferência e aceitação do consumidor. Além disso, de acordo com Coradin et al. (2011), um dos aspectos a ser considerado para uma planta apícola é sua capacidade de possuir intensa floração e por longo período, principalmente em épocas de maior escassez de recursos florais. Nesse estudo não foram consideradas as datas de início e final de floração de cada planta, principalmente por ainda serem desconhecidas as espécies que floresciam em cada local. Ainda, eram esperadas uma diversidade florística e variações sazonais nos ciclos de floração devido às diferenças climáticas e aos diferentes tipos de relevos (planaltos, serras, vales, entre outros) das regiões amostradas. Dessa forma, foram realizadas três coletas com intervalos de aproximadamente 20 dias (Tabela 1) para poder avaliar a disponibilidade e diversidade de espécies com potencial melífero. O monitoramento do ciclo de floração de espécies que estão próximas da colmeia pode dar uma estimativa de quais plantas contribuíram para a produção do mel, uma vez que esse produto apícola é classificado (unifloral ou multifloral) conforme a origem da matéria prima utilizada na sua produção (Brasil, 2000). De acordo com as informações apresentadas na Tabela 3, Sphagneticola trilobata, Trifolium repens, T. pratense, Cajanus cajan, Vicia sp., Verbena litoralis, Lantana undulata, Raphanus sativus e Clidemia hirta permaneceram com flores entre o primeiro e último dia de coleta ( 60 dias) em quatro (Joinville, Caçador, Araranguá e São Joaquim) das seis zonas agroecológicas, as quais também foram regiões com maior número de amostras coletadas e identificadas (Figura 2).

Além da identificação de espécies com potencial apícola, informações importantes também foram relatadas a respeito da estimativa da distância percorrida pelas abelhas (Apis mellifera) entre a colmeia e as plantas de cada região desse estudo. As abelhas possuem alta capacidade de forragear em uma distância aproximada de até 3km ao redor do apiário (Camargo, 2002). Nesse estudo, a estimativa de distâncias de voo variou entre 8 e 1154 m, geralmente não ultrapassando 750 m (Tabela 3 ). As distâncias apresentaram maior variabilidade para as amostras provenientes de Araranguá e Caçador, respectivamente de 86 a $1108 \mathrm{~m}$ e de 11 a 1154 m, enquanto foram menos variáveis para as de Joinville e Mafra, sendo de 8 a 523 m e de 34 a 436 m, respectivamente. Para as amostras de São Joaquim e São Miguel do Oeste as distâncias variaram de 22 a 516 m e de 222 a 830 $\mathrm{m}$, respectivamente. De maneira geral, as abelhas coletam pólen em um raio de $2 \mathrm{~km}$ em torno da colmeia, mas podem voar entre 5 e 13km para procurar outras fontes (Coradin, et al., 2011). Conforme relatado por Camargo (2002) quanto menor a distância entre colmeia e flora apícola, maior será a produção de mel, uma vez que o transporte do pólen e do néctar será realizado em menor tempo e com menor gasto energético das abelhas nos deslocamentos. O mesmo autor ressalta que, além da distância, o aspecto produtivo é diretamente influenciado pela qualidade (variedade e densidade populacional das espécies e diferentes períodos de floração) das floradas.

Os resultados desse estudo indicam a necessidade de um plano de monitoramento de vegetações que se desenvolvem próximas de sistemas de produção apícola para auxiliar, não somente os apicultores a encontrarem fontes adequadas e de abundante suprimento de alimentos às abelhas, mas também os agentes técnicos e comunidade científica de forma a evitar informações errôneas. Por meio do registro contínuo de vegetações também será possível identificar o surgimento de novas espécies, contribuir para a conservação da flora nativa, bem como contribuir para o setor apícola e para pesquisadores de diversas áreas por meio do fornecimento desses dados.

As ações de caracterização e monitoramento de plantas com potencial apícola também poderão auxiliar a comunidade científica e órgãos regulamentadores de alimentos em relação à presença de compostos químicos em produtos apícolas, como os alcaloides pirrolizidínicos (AP). Esses compostos são toxinas naturais produzidas por alguns gêneros de Asteraceae, Fabaceae e Boraginaceae (Bandini, et al., 2020) e são comumente encontrados em produtos apícolas, principalmente no mel e no pólen (Brugnerotto et al., 2021). Portanto, a presença de AP é considerada indesejável, principalmente se a soma desses compostos estiver acima de $71 \mu \mathrm{g} \mathrm{kg}^{-1}$ (EFSA, et al., 2017) em méis e $500 \mu \mathrm{g} \mathrm{kg}^{-1}$ em polens (European Commission, 2020). 
Considerando que nesse trabalho as famílias Asteraceae e Fabaceae foram predominantes, é recomendando que os produtos apícolas provenientes das regiões estudadas sejam avaliados em relação ao conteúdo de AP.

\section{Considerações Finais}

Esse foi o primeiro estudo que coletou e identificou espécies de plantas com potencial apícola em sistemas de monitoramento digital apícola no estado de Santa Catarina. Mesmo sendo resultados exploratórios e com limitações principalmente em relação ao baixo número de amostras e curto período de coleta das plantas -, foi possível identificar uma pequena parte da flora com potencial apícola em espaços específicos em seis zonas agroecológicas, as quais representam as principais mesorregiões de Santa Catarina e que também são produtoras de mel. Também foi possível estimar a distância que as abelhas percorreriam para coletar os recursos nectaríferos ou polínicos devido ao registro da localização de cada planta coletada. Alguns fatores, entre eles, a diferença climática e de relevos, podem ser os principais responsáveis pela heterogeneidade das plantas com potencial apícola identificadas nesse estudo, representadas pelas 81 espécies encontradas. Essa diversidade caracteriza o estado de Santa Catarina com excelentes condições para exploração da atividade apícola, a qual contribui não somente para produção de diversos produtos apícolas, mas também para a produção de frutas e grãos por meio da polinização, na diversificação cultural para agricultores, bem como, para a conservação das vegetações. É importante destacar que, a partir das informações obtidas nesse estudo, o levantamento das pastagens apícolas geralmente será regional, justamente pela variabilidade de clima e relevo que alteram o fluxo de produção de néctar e pólen (épocas de floração). Devido a isso, é fundamental que os agentes técnicos e pesquisadores juntamente com os apicultores desenvolvam um plano de monitoramento de floradas com calendário de floração próprio para cada região de produção, acompanhando o início e término das floradas em relação às mudanças climáticas. Esse plano também pode ser uma estratégia interessante para introduzir nos apiários plantas com capacidade adaptativa ao clima e relevo das regiões, com períodos de floração diferenciados para proporcionar oferta contínua de néctar e pólen às abelhas e maximizar a produção de mel e outros produtos apícolas. O conjunto dessas informações deixará de ser um conhecimento empírico e passará a contribuir para a conservação da vegetação nativa, da exploração sustentável das espécies e das abelhas, para o fortalecimento da cadeia produtiva de mel e de monitoramento das colmeias.

\section{Agradecimentos}

Aos órgãos de fomento Fundação de Amparo à Pesquisa e Inovação do Estado de Santa Catarina (FAPESC) (no. 88887.178578/2018-00) e Coordenação de Aperfeiçoamento de Pessoal de Nível Superior (CAPES) (código 001).

\section{Referências}

ABEMEL - Associação Brasileira dos Exportadores de de Mel. (2020). Dados Estatísticos do Mercado de Mel 2016 a 2019. Brazil lets bee. https://www.brazilletsbee.com.br/Dados Estatísticos do Mercado de Mel_2016_a_2019_11nov2020.pdf

Almeida, D. De, Marchini, L. C., Sodré, G. D. S., D’Ávila, M., \& Arruda, C. M. F. de. (2003). Plantas visitadas por abelhas e polinização. Série Produtor Rural. ESALQ - Divisão de Biblioteca e Documentação.

Bandini, T. B., Siani, A. C., \& Spisso, B. F. (2020). A botanical census on pyrrolizidine alkaloid-producing species in Brazilian herbaria: data set for a potential health risk indication. Rodriguésia, 71, 1-19. https://doi.org/10.1590/2175-7860202071118

Bergamo, G. (2019). Marcadores de autenticidade para diferenciação entre méis de melato de bracatinga (Mimosa scabrella Bentham) e florais produzidos no estado de Santa Catarina. Universidade Federal de Santa Catarina.

Brasil. (2000). Instrução Normativa n ${ }^{\circ} 11$, de 20 de outubro de 2000. Aprova Regulamento técnico de Identidade e Qualidade do Mel. In MAPA- Ministério da Agricultura, Pecuária e Abastecimento (pp. 1-4).

Brugnerotto, P., Seraglio, S. K. T., Schulz, M., Gonzaga, L. V., Fett, R., \& Costa, A. C. O. (2021). Pyrrolizidine alkaloids and beehive products: A review. Food Chemistry, 342, 128384. https://doi.org/10.1016/j.foodchem.2020.128384 
Camargo, R. C. R. de. (2002). Sistemas de Produção - Produção de mel. (E. Meio-Norte, Ed.). https://doi.org/0104-866X

Carvalho, C. A. L. de, \& Marchini, L. C. (1999). Plantas visitadas por Apis mellifera L. no vale do rio Paraguaçu, Município de Castro Alves, Bahia. Revista Brasileira de Botânica, 22, 333-338. https://doi.org/10.1590/s0100-84041999000500016

Coradin, L., Siminski, A., \& Reis, A. (2011). Espécies nativas da flora brasileira de valor econômico atual ou potencial plantas para o futuro - Região Sul.

EFSA, CONTAM, P. C. F. C., Knutsen, H. K., Alexander, J., Barregård, L., Bignami, M., Binaglia, M. (2017). Risks for human health related to the presence of pyrrolizidine alkaloids in honey, tea, herbal infusions and food supplements. EFSA Journal, 15(7). https://doi.org/10.2903/j.efsa.2017.4908

Epagri - Empresa de Pesquisa Agropecuária e Extensão Rural de Santa Catarina (2021). Apis On-line. Plataforma Apis-Online. https://ciram.epagri.sc.gov.br/apicultura/apresentacao.html

European Commission. (2020). Annex to the Comission Regulation (EU) .../... amending Regulation (EC) 1881/2006 as regards maximum levels of pyrrolizidine alkaloids in certain foodstuffs. European Comission. https://doi.org/10.1017/CBO9781107415324.004

IBGE - Instituto Brasileiro de Geografía e Estatística. (2017). Tabela 6935 - Número de estabelecimentos agropecuários com apicultura e venda de produtos. Sidra. https://sidra.ibge.gov.br/tabela/6935

Köppen, W. (1948). Climatologia: con un estudio de los climas de la tierra. Fondo de Cultura Econômica. México. Fondo de Cultura Economica.

Nishida, S. M., Naide, S. S., \& Pagnin, D. (2014). Plantas que atraem aves e outros bichos. Editora UNESP. https://repositorio.unesp.br/bitstream/handle/11449/126246/ISBN9788579835391.pdf?sequence=1\&isAllowed=y

ONU - Organização das Nações Unidas. (2021). Agenda 2030 para o desenvolvimento sustentável. Plataforma Agenda 2030. http://www.agenda2030.org.br/sobre/

Pirani, J. R., \& Cortopassi-Laurino, M. (1993). Flores e abelhas em São Paulo. USP \& FAPESP.

Reis, A. (1996-2013). Flora Ilustrada Catarinense. Herbário Barbosa Rodrigues, Itajaí.

Reitz, R. (1965- 1989). Flora Ilustrada Catarinense. Herbário Barbosa Rodrigues, Itajaí.

Sá, R. O. de. (2005). Variabilidade genética entre progênies de meios irmãos de nabo forrageiro (Raphanus sativus L. var. oleiferus) cultivar Cati Al 1000. Universidade Estadual Paulista Júlio de Mesquita Filho.

Salomé, J. A. (2002). Levantamento e fenologia de plantas apícolas do estado de Santa Catarina. Universidade Federal de Santa Catarina.

Salomé, J. A., \& Orth, A. I. (2004). Diversidade da flora apícola de Santa Catarina. Agropecuária Catarinense, 17(2), 84-88.

Santos, R., Zanette, V. C., Elias, G. A., \& Padilha, P. T. (2016). Biodiversidade em Santa Catarina: Parque estadual da Serra Furada. (D. de O. Estevam \& Revisão, Eds.), Ediunesc (Vol. 3). Criciúma: Biblioteca Central Prof. Eurico Back - UNESC. Retrieved from http://www.unesc.net/portal/capa/índex/ $300 / 5886 /$

Santos, R. F., Helena, L., Kiill, P., P. E. Semi-árido, Lincoln, J., Araújo, P., \& Cep, P. E. S. (2006). Levantamento da flora melífera de interesse apícola no Mmunicípio de Petrolina-Pe. Revista Caatinga, 19(3), 221-227.

Silva, P. M. (2016). Caracterização e estabilidade de compostos químicos em méis de abelhas Apis mellifera L. produzidos no estado de Santa Catarina. Universidade Federal de Santa Catarina.

Silva, M. (2005). Abelhas e plantas melíferas da zona rural dos municípios de Cocal do Sul, Criciúma e Nova Veneza, situados na região carbonífera no sul do estado de Santa Catarina. Universidade do Extremo Sul Catarinense, Criciúma.

Souza, V.C., \& Lorenzi, H. (2019). Botânica sistemática. Guia ilustrado para identificação das famílias de Fanerógamas nativas e exóticas no Brasil, baseado em APG IV. (4ed. Instituto Plantarum).

Stuessy, T. F. (2009). Plant taxonomy. The systematic evaluation of comparative data. Columbia. University Press.

Thomé, V. M. R., Zampieri, S. ., Braga, H. J. ., Pandolfo, C. ., Silva Júnior, V. P. da. ., Laus Neto, J.; Soldateli, D.; Gebler, E.; Ore, J. D.; Echeverria, L. ., ... Suski, P. P. (1999). Zoneamento Agroecológico e Socioeconômico de Santa Catarina. Florianópolis. Epagri.

Vibrans, A. C., Sevegnani, L., Gasper, A. L. de, \& Lingner, D. V. (2013). Floresta Ombrófila Mista. Inventário Florístico Florestal de Santa Catarina. Universidade Regional de Blumenau. Editora 